\title{
Application of global Carleman estimates with rotated weights to an inverse problem for the wave equation
}

\author{
Anna Doubova ${ }^{\mathrm{a}}$, Axel Osses ${ }^{\mathrm{b}}$ \\ ${ }^{a}$ Dpto. E.D.A.N., University of Sevilla, Aptdo. 1160, 41080 Sevilla, Spain. Tel: (34)954557993, fax: (34)954552898 \\ b Dpto. de Ingenería Matemática, University of Chile, Casilla 170/3, correo 3, Santiago, Chili. Tel: (562)6784994, \\ fax: (562)6883821
}

\begin{abstract}
We establish geometrical conditions for the inverse problem of determining a stationary potential in the wave equation with Dirichlet data from a Neumann measurement on a suitable part of the boundary. We present the stability results when we measure on a part of the boundary satisfying a rotated exit condition. The proofs rely on global Carleman estimates with angle type dependence in the weight functions. To cite this article: A. Doubova, A. Osses, C. R. Acad. Sci. Paris, Ser. I 340 (2005).

\section{Résumé}

Application d'une inégalité de Carleman globale avec des poids à direction variable à un problème inverse pour l'équation des ondes. On établit des conditions geométriques pour le problème inverse consistant à déterminer un potentiel stationnaire dans l'équation des ondes avec une donnée de Dirichlet et à partir d'une mesure de Neumann sur une partie appropriée de la frontière. On présente des résultats de stabilité quand on mesure sur une partie de la frontière satisfaisant une condition sortante à directions variables. Les démonstrations réposent sur des inégalités de Carleman globales avec des fonctions poids qui dépendent d'un angle comme paramètre. Pour citer cet article: A. Doubova, A. Osses, C. R. Acad. Sci. Paris, Ser. I 340 (2005).
\end{abstract}

\section{Version française abrégée}

Soient $\Omega$ un ouvert borné de $\mathbb{R}^{2}$ de frontière $\partial \Omega$ de classe $C^{2}, \Gamma_{0}$ une partie ouverte non vide de $\partial \Omega$, $\nu$ la normale unitaire extérieure à $\Omega, T>0$ et $\mathcal{U}_{M}=\left\{q \in L^{\infty}(\Omega):\|q\|_{L^{\infty}(\Omega)} \leq M\right\}$, où $M>0$ est une constante. On s'intéresse à la stabilité du problème inverse suivant: Etant donné $u_{0}, u_{1}, h$ et $\xi$ dans des

Email addresses: doubova@us.es (Anna Doubova), axosses@dim.uchile.cl (Axel Osses). 
espaces appropriés, on cherche des conditions suffisantes sur $\Gamma_{0}$ et $T$ telles qu'il existe $q \in \mathcal{U}_{M}$ tel que la solution u de l'équation des ondes

$$
\begin{cases}\partial_{t t} u-\Delta u+q(x) u=0 & \text { dans } \Omega \times(0, T), \\ u=h & \text { sur } \partial \Omega \times(0, T), \\ u(x, 0)=u_{0}, \quad \partial_{t} u(x, 0)=u_{1} & \text { dans } \Omega\end{cases}
$$

satisfait la condition additionnelle

$$
\frac{\partial u}{\partial \nu}=\xi \quad \text { sur } \Gamma_{0} \times(0, T)
$$

On dénote par $u$ et $\bar{u}$ les solutions de (1) associées à $q$ et à un potentiel connu $\bar{q}$ respectivement. Si $f=q-\bar{q}$ et $y=u-\bar{u}$ alors $q u-\bar{q} \bar{u}=q y+f \bar{u}$ et le problème inverse correspondant pour la fonction $y$ est : Etant donné $q \in \mathcal{U}_{M}, R=-\bar{u}$ et $\bar{\xi}=\frac{\partial \bar{u}}{\partial \nu}$ dans des espaces appropriés, on cherche des conditions suffisantes sur $\Gamma_{0}$ et $T$ telles qu'il existe une fonction indépendante du temps $f$ telle que la solution y de

$$
\begin{cases}\partial_{t t} y-\Delta y+q(x) y=f(x) R(x, t) & \text { dans } \Omega \times(0, T), \\ y=0 & \text { sur } \partial \Omega \times(0, T), \\ y(x, 0)=\partial_{t} y(x, 0)=0 & \text { dans } \Omega\end{cases}
$$

satisfait la condition additionnelle

$$
\frac{\partial y}{\partial \nu}=\xi-\bar{\xi} \quad \text { sur } \Gamma_{0} \times(0, T)
$$

Dans [9] et [5] on démontre des résultats de stabilité quand $\Gamma_{0}$ inclut la région sortante (voir [7]) de la frontière par rapport à un certain point $x_{0}$ (voir Figure 1 gauche). Dans cette Note on étend les hypothèses géométriques précédentes au sens qu'il suffit que $\Gamma_{0}$ contienne une partie de la frontière satisfaisant une condition décrite par un paramètre d'angle $\theta \in(-\pi / 2, \pi / 2)$, voir (12). Si $\theta=0$ on récupère la condition sortante précédente. Pour $\theta \rightarrow \pi / 2$ ou $\theta \rightarrow-\pi / 2$ on obtient par exemple des conditions plus générales (voir Figure 1 centre).

Théorème 0.1 On suppose que $\Gamma_{0}$ vérifie (12). On considère $u$ et $\bar{u}$ les solutions de (1) associées à $q \in$ $\mathcal{U}_{M}$ et à un potentiel connue $\bar{q} \in L^{\infty}(\Omega)$ respectivement et avec des mesures $\xi$ données par $(2)$ et $\bar{\xi}=\frac{\partial \bar{u}}{\partial \nu}$. Il existe un temps $T_{1}=T_{1}\left(\Omega, \theta, x_{0}\right)>0$ tel que si $T>T_{1}, \bar{u} \in H^{1}\left(0, T ; L^{\infty}(\Omega)\right)$ et $|\bar{u}(x, 0)| \geq \alpha_{0}>0$ presque partout dans $\Omega$, alors il existe une constante $C=C\left(T, M,\|\bar{q}\|_{L^{\infty}},\|\bar{u}\|_{H^{1}\left(L^{\infty}\right)}, \alpha_{0}\right)>0$ telle que

$$
\|q-\bar{q}\|_{L^{2}(\Omega)} \leq C\|\xi-\bar{\xi}\|_{H^{1}\left(0, T ; L^{2}\left(\Gamma_{0}\right)\right)} \quad \forall q \in \mathcal{U}_{M}
$$

La preuve de ce résultat est un cas particulier du théorème suivant. Il suffit de prendre $f=q-\bar{q}$ et d'utiliser le fait que la constante de stabilité dans (6) ne dépend que de $M$.

Théorème 0.2 On suppose que $\Gamma_{0}$ et $T$ vérifient les hypothèses du Théorème 0.1 . Soient $f \in L^{2}(\Omega)$, $R \in H^{1}\left(0, T ; L^{\infty}(\Omega)\right)$ avec $\left\|\partial_{t} R\right\|_{L^{2}\left(L^{\infty}\right)} \leq M_{1}$ et $|R(x, 0)| \geq \alpha_{0}>0$ presque partout dans $\Omega$. Alors, il existe une constante $C=C\left(T, M, M_{1}, \alpha_{0}\right)>0$ telle que si $y$ est la solution de (3) on a

$$
\|f\|_{L^{2}(\Omega)} \leq C\left\|\frac{\partial y}{\partial \nu}\right\|_{H^{1}\left(0, T ; L^{2}\left(\Gamma_{0}\right)\right)} \quad \forall q \in \mathcal{U}_{M} .
$$

La démonstration est basée sur une inégalité globale de Carleman pour l'équation des ondes (Proposition 2.1). On utilise aussi les arguments de [5,9] pour l'appliquer au problème inverse. Les démonstrations détaillées des théorèmes précédents seront données dans [2]. 


\section{Introduction and main results}

Let $\Omega$ be a bounded open subset of $\mathbb{R}^{2}$ with boundary $\partial \Omega$ of class $C^{2}, \Gamma_{0}$ a nonempty open subset of $\partial \Omega, \nu$ the unit outward normal vector to $\Omega, T>0$ and $\mathcal{U}_{M}=\left\{q \in L^{\infty}(\Omega):\|q\|_{L^{\infty}(\Omega)} \leq M\right\}$, where $M>0$ is a constant. We are interested in the stability of the following inverse problem: Given $u_{0}, u_{1}$, $h$ and $\xi$ in appropriate spaces, we look for sufficient conditions under $\Gamma_{0}$ and $T$ such that we can find $q \in \mathcal{U}_{M}$ such that the solution $u$ of the wave equation

$$
\begin{cases}\partial_{t t} u-\Delta u+q(x) u=0 & \text { in } \Omega \times(0, T), \\ u=h & \text { on } \partial \Omega \times(0, T), \\ u(x, 0)=u_{0}, \quad \partial_{t} u(x, 0)=u_{1} & \text { in } \Omega\end{cases}
$$

satisfies the additional condition

$$
\frac{\partial u}{\partial \nu}=\xi \quad \text { on } \Gamma_{0} \times(0, T)
$$

Let us denote by $u$ and $\bar{u}$ the solutions of (7) associated to $q$ and to some known potential $\bar{q}$ respectively. If we set $f=q-\bar{q}$ and $y=u-\bar{u}$ then $q u-\bar{q} \bar{u}=q y+f \bar{u}$ and we obtain the following equivalent inverse problem for the function $y$ : Given $q \in \mathcal{U}_{M}, R=-\bar{u}$ and $\bar{\xi}=\frac{\partial \bar{u}}{\partial \nu}$ in appropriate spaces, we look for sufficient conditions for $\Gamma_{0}$ and $T$ such that we can find a time independent function $f$ such that the solution $y$ of

$$
\begin{cases}\partial_{t t} y-\Delta y+q(x) y=f(x) R(x, t) & \text { in } \Omega \times(0, T), \\ y=0 & \text { on } \partial \Omega \times(0, T), \\ y(x, 0)=\partial_{t} y(x, 0)=0 & \text { in } \Omega\end{cases}
$$

satisfies the additional condition

$$
\frac{\partial y}{\partial \nu}=\xi-\bar{\xi} \quad \text { on } \Gamma_{0} \times(0, T)
$$

Let $a>0, b \in \mathbb{R}$ with $a^{2}+b^{2}=1$ and

$$
\theta=\tan ^{-1}(b / a) \in(-\pi / 2, \pi / 2), \quad a=\cos \theta, \quad b=\sin \theta .
$$

The main novelty here is that we can consider $\Gamma_{0}$ sufficiently large in such a way that

$$
\exists x_{0} \in \mathbb{R}^{2} \backslash \bar{\Omega} \text { such that } \Gamma_{0} \supseteq\left\{x \in \partial \Omega:\left(x-x_{0}\right) \cdot(a I-b A) \nu(x)>0\right\},
$$

where $A=\left(a_{i j}\right), i, j \in\{1,2\}, a_{11}=a_{22}=0, a_{12}=-1, a_{21}=1$ and $I$ is the identity matrix. Notice that $(a I-b A) \nu(x)$ corresponds to a clockwise rotation of the normal field in an angle $\theta$. In [9] and [5] the stability results were obtained when $\Gamma_{0}$ includes the exit boundary region with respect to some exterior point $x_{0}$ (see Figure 1 left). In this Note we extend this geometrical hypothesis in the sense that it suffices that $\Gamma_{0}$ contains a rotated exit part of the boundary which can be described by the angle parameter $\theta$. For $\theta=0$ we recover the exit boundary region (see Figure 1 left). For $\theta \rightarrow \pi / 2$ and $\theta \rightarrow-\pi / 2$ we obtain for example more general geometrical conditions (see Figure 1 center). There are of course an infinite number of intermediate cases for $\theta \in(-\pi / 2, \pi / 2)$. This geometrical condition is only sufficient in order to solve the inverse problem. A reasonable conjecture is that it is only necessary to measure in order to capture the geometrical optic rays (see [1]) coming from the support of the unknown potential. In fact, 
a generic stability can be obtained (see [10]) for the same inverse problem considered in this Note under this weaker condition.
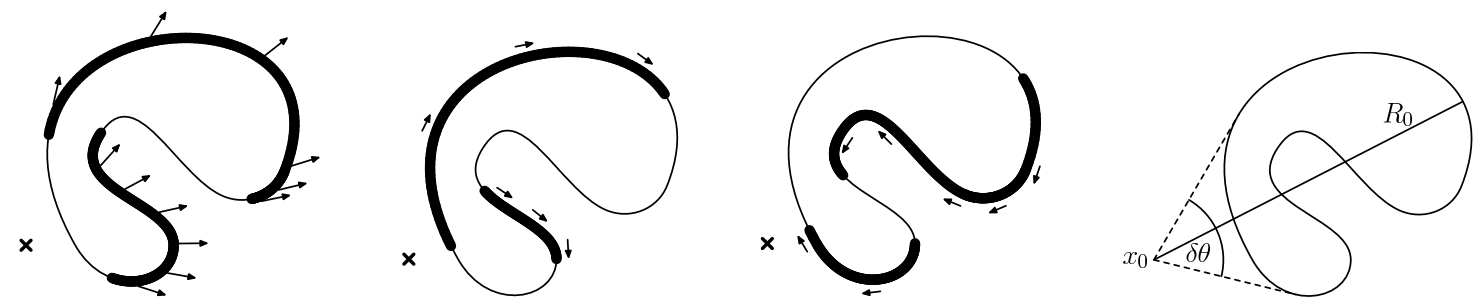

Figure 1. From left to right: rotated exit boundary regions in a domain corresponding to $\theta=0, \theta \rightarrow \pi / 2$ and $\theta \rightarrow-\pi / 2$ with respect to some exterior point $x_{0}$. The last figure shows the main geometrical parameters of the problem.

Figure 1. De gauche à droite: les régions de la frontière satisfaisant une condition sortante à directions variables correspondante à $\theta=0, \theta \rightarrow \pi / 2$ et $\theta \rightarrow-\pi / 2$ par rapport à un point extérieur $x_{0}$. La dernière figure montre les principaux paramètres géométriques du problème.

Theorem 1.1 Suppose that $\Gamma_{0}$ satisfies (12). Let $u$ and $\bar{u}$ be the respective solutions of (7) associated to $q \in \mathcal{U}_{M}$ and $\bar{q} \in L^{\infty}(\Omega)$ with measurements $\xi$ given by (8) and $\bar{\xi}=\frac{\partial \bar{u}}{\partial \nu}$. There exists a time $T_{1}=T_{1}\left(\Omega, \theta, x_{0}\right)>0$ such that if $T>T_{1}, \bar{u} \in H^{1}\left(0, T ; L^{\infty}(\Omega)\right)$ and $|\bar{u}(x, 0)| \geq \alpha_{0}>0$ a.e. in $\Omega$, then there exists a constant $C=C\left(T, M,\|\bar{q}\|_{L^{\infty}},\|\bar{u}\|_{H^{1}\left(L^{\infty}\right)}, \alpha_{0}\right)>0$ such that

$$
\|\bar{q}-q\|_{L^{2}(\Omega)} \leq C\|\bar{\xi}-\xi\|_{H^{1}\left(0, T ; L^{2}\left(\Gamma_{0}\right)\right)} \quad \forall q \in \mathcal{U}_{M}
$$

Notice that this result is of a local nature, since the stability holds around some $\bar{q} \in L^{\infty}(\Omega)$ for which the corresponding solution $\bar{u}$ of problem (7) is regular enough. The proof of the Theorem 1.1, in view of the previous discussions, is a particular case of the following result. It is sufficient to take $f=q-\bar{q}$ and to use the fact that the stability constant in (14) depends only on $M$.

Theorem 1.2 Assume $\Gamma_{0}$ and $T$ verify the hypothesis of Theorem 1.1. Let be given $f \in L^{2}(\Omega), R \in$ $H^{1}\left(0, T ; L^{\infty}(\Omega)\right)$ with $\left\|\partial_{t} R\right\|_{L^{2}\left(L^{\infty}\right)} \leq M_{1}$ and $|R(x, 0)| \geq \alpha_{0}>0$ a.e. in $\Omega$. Then, there exists a constant $C=C\left(T, M, M_{1}, \alpha_{0}\right)>0$ such that if $y$ is the solution of (9) we have

$$
\|f\|_{L^{2}(\Omega)} \leq C\left\|\frac{\partial y}{\partial \nu}\right\|_{H^{1}\left(0, T ; L^{2}\left(\Gamma_{0}\right)\right)} \quad \forall q \in \mathcal{U}_{M} .
$$

The proof of this theorem will be sketched in Section 3. It is based on the obtention of a global Carleman estimate with a modified weight given in Proposition 2.1. We are able to construct the appropiate weight function only in two dimensions. More details will be given in a forthcoming article [2], where an explicit expression for the inversion time $T_{1}$ is given. We make here only two remarks about this time:

Remark 1 As $\theta \rightarrow \pm \frac{\pi}{2}$, we have $a \rightarrow 0$ and $b \rightarrow \pm 1$ and the inversion time $T_{1} \rightarrow+\infty$. More precisely

$$
\begin{gathered}
T_{1} \approx \exp (\delta \theta|\tan \theta|)\left(R_{0}^{2} \exp (\delta \theta|\tan \theta|)-r_{0}^{2}\right)^{1 / 2}, \\
R_{0}=\sup _{x \in \Omega}\left|x-x_{0}\right|, \quad r_{0}=\inf _{x \in \Omega}\left|x-x_{0}\right|, \quad \delta \theta=\sup _{x \in \Omega} \arg \left(x-x_{0}\right)-\inf _{x \in \Omega} \arg \left(x-x_{0}\right) .
\end{gathered}
$$

This fact recalls the one encountered in [8] where the exact controllability time for the wave equation with a rotated boundary control region was of order $\frac{2 R_{0}}{\cos \theta}$ as $\theta \rightarrow \pm \frac{\pi}{2}$.

Remark 2 In the case $\theta=0$ we also recover $T_{1}=\left(R_{0}^{2}-r_{0}^{2}\right)^{1 / 2} / \sqrt{\beta}$, where the constant $\beta$ is introduced in the next section (see [5,9] and the idea for a better estimation of this time in [6]).

Remark 3 The hypothesis $x_{0} \notin \bar{\Omega}$ in (12) is maybe not necessary in Theorems 1.1 and 1.2 (see [2]). 


\section{Global Carleman inequality with rotated weights}

Let us set $Q=\Omega \times(-T, T), \Sigma_{0}=\Gamma_{0} \times(-T, T)$. Given $\beta \in\left(0, \beta_{2}\right)$ with $\beta_{2}=\beta_{2}\left(a, b, \theta, x_{0}\right)$, we introduce the following weight function:

$$
\Phi(x, t)=a \phi(x) \exp (2 a \widehat{\phi}(x) / b)-\beta t^{2}, \quad \varphi(x, t)=\exp (\lambda \Phi(x, t)) \quad x \in \bar{\Omega}, t \in \mathbb{R}, \lambda>0,
$$

where $\phi$ and $\widehat{\phi}$ are given by

$$
\phi(x)=\left|x-x_{0}\right|^{2}, \quad \widehat{\phi}(x)=\arg \left(x-x_{0}\right) .
$$

Proposition 2.1 Assume that $\Gamma_{0}$ satisfies (12). Then for all $M>0$, there exist positive constants $\bar{\lambda}, \bar{\mu}$ and $C$ depending on $a, b, \Omega$ and $x_{0}$ such that for any $q \in L^{\infty}(Q)$ with $\|q\|_{L^{\infty}(Q)} \leq M$, for any $\lambda \geq \bar{\lambda}$ and for any $s \geq \bar{s}=\exp \left(\bar{\mu} \lambda\left(1+T^{2}\right)\right) M^{2 / 3}$ the following estimate holds:

$$
\begin{aligned}
& s \lambda \iint_{Q} e^{2 s \varphi} \varphi\left(\left|\partial_{t} v\right|^{2}+|\nabla v|^{2}\right) d x d t+s^{3} \lambda^{3} \iint_{Q} e^{2 s \varphi} \varphi^{3}|v|^{2} d x d t \\
& \leq C\left(\iint_{Q} e^{2 s \varphi}\left|\partial_{t t} v-\Delta v+q(x, t) v\right|^{2} d x d t+s \lambda \iint_{\Sigma_{0}} e^{2 s \varphi}\left|\frac{\partial v}{\partial \nu}\right|^{2} d \sigma d t\right)
\end{aligned}
$$

for all $v \in C^{2}(\bar{\Omega} \times[-T, T])$ such that $v=0$ on $\Sigma, v(x, \pm T)=0$ and $\partial_{t} v(x, \pm T)=0$.

Sketch of the proof.- We follow the method from [4] which involves a lot of computations not given here. Nevertheless, let us precise some important things. Let us notice first that the properties of the weight function $\Phi$ given in (17) are in narrow connection with the geometrical conditions (12) on $\Gamma_{0}$. Indeed, after splitting the corresponding conjugate operator, it appears the adjoint equation multiplied by the gradient of the solution following some direction $\nabla \Phi$. Taking the idea from [8], we choose $\Phi$ with a gradient $\nabla \Phi$ which is essentially a rotation of the original field $\left(x-x_{0}\right)$, but with a magnitude which radially depends in space:

$$
\nabla \Phi=(a \nabla \phi+2 b \phi \nabla \widehat{\phi}) \exp (2 a \widehat{\phi} / b)=2(a I+b A)\left(x-x_{0}\right) \exp (2 a \widehat{\phi} / b) .
$$

Secondly, in order to have (19), the weight function $\Phi$ must satisfy in addition other algebraic conditions which are obtained with a carefully choice of $\beta_{2}$.

Remark 4 Notice that in (19) the source term $q=q(x, t)$ can depend on $x$ and $t$ with a constant depending uniformly on $q$. On the other hand (19) does not require any sufficiently large size for the time interval $2 T$.

\section{Application to the inverse problem}

Let us present the main steps of the proof of Theorem 1.2. We use the arguments of [5,9]. First, we set $\psi=\partial_{t} y$ where $y$ is the solution of (9). Then, we have

$$
\begin{cases}\partial_{t t} \psi-\Delta \psi+q(x) \psi=f(x) \partial_{t} R(x, t) & \text { in } \Omega \times(0, T), \\ \psi=0 & \text { on } \partial \Omega \times(0, T), \\ \psi(x, 0)=0, \quad \partial_{t} \psi(x, 0)=f(x) R(x, 0) & \text { in } \Omega .\end{cases}
$$

Thanks to the hypothesis of Theorem 1.2 on $f, R$ and $q$ and using the regularity properties of the wave equation (see for example [3]) we deduce that $\psi \in C\left([0, T] ; H_{0}^{1}(\Omega)\right) \cap C^{1}\left([0, T] ; L^{2}(\Omega)\right)$, $\frac{\partial \psi}{\partial \nu} \in$ 
$L^{2}\left(0, T ; L^{2}(\partial \Omega)\right)$. Thus, we obtain that $y \in C\left([0, T] ; H_{0}^{1}(\Omega) \cap H^{2}(\Omega)\right) \cap C^{1}\left([0, T] ; H_{0}^{1}(\Omega)\right) \cap C^{2}\left([0, T] ; L^{2}(\Omega)\right)$. Extending $\partial_{t} R$ and $\psi$ on $\Omega \times(-T, 0)$ as even functions and calling the extensions by the same symbols, we obtain that $y$ is the solution of (9) defined now in $\Omega \times(-T, T)$ with the regularity as before.

In order to apply the Carleman estimate (19), we introduce

$$
z=e^{s \varphi} \chi \partial_{t} y
$$

where $\chi \in C_{c}^{\infty}([-T, T]), 0 \leq \chi \leq 1$ is a suitable cut-off function and $\varphi$ is given by (17). Notice that $z(x, \pm T)=\partial_{t} z(x, \pm T)=0$ and then after some computations we get

$$
\frac{1}{2} \int_{\Omega}|f(x)|^{2}|R(x, 0)|^{2} e^{2 s \varphi(x, 0)} d x=\int_{-T \Omega}^{0} \int_{\Omega}\left(\partial_{t t} z-\Delta z+q(x) z\right) \partial_{t} z d x d t .
$$

In the last step, we use Carleman inequality (19) in order to estimate the right hand side of (21) in terms of $\left\|\frac{\partial y}{\partial \nu}\right\|_{H^{1}\left(0, T ; L^{2}\left(\Gamma_{0}\right)\right)}$. At this point, we also use that $\varphi(x, \pm T)<\varphi(x, 0)$ uniformly for all $x \in \bar{\Omega}$ whenever $T>T_{1}$ and that $|R(x, 0)| \geq \alpha_{0}>0$. This gives (14) and to conclude the proof of Theorem 1.2.

\section{Acknowledgements}

The authors acknowledge J.-P. Puel for fruitful discussions about the subject. The first author was partially supported by D.G.E.S. grant BFM2003-06446. The second author was partially supported by FONDAP in Applied Mathematics and by grants FONDECYT 1030808, ECOS CO1E02-CO4E08 and INRIA-CONICYT. This Note has been finished during the stay of the second author at the Jacques-Louis Lions Laboratory at the University Pierre et Marie Curie - Paris 6, and the stay was supported by the CNRS.

\section{References}

[1] C. Bardos, G. Lebeau, and J. Rauch, Sharp sufficient conditions for the observation, control and stabilization of waves from the boundary, SIAM J. Contr. Optim., 30 (1992) 1024-1465.

[2] A. Doubova, A. Osses, Rotated weights in global Carleman estimates applied to an inverse problem for the wave equation, to appear.

[3] R. Dautray, J.-L. Lions, Mathematical analysis and numerical methods for science and technology, Springer-Verlag, Berlin, 2000.

[4] O. Yu. Imanuvilov, On Carleman estimates for hyperbolic equations, Asymptot. Anal. 32 (3-4) (2002) 185-220.

[5] O. Yu. Imanuvilov, M. Yamamoto, Global uniqueness and stability in determining coefficients of wave equations, Commun. Partial Diff. Eqns 26 (7-8) (2001) 1409-1425.

[6] O. Yu. Imanuvilov, M. Yamamoto, Determination of a coefficient in an acoustic equation with a single measurement, Inverse Problems, 19 (2003) 157-171.

[7] J.-L. Lions, Contrôlabilité exacte, perturbation et stabilisation de Systèmes Distribués, 1, Masson, Paris, 1988.

[8] A. Osses, A rotated multiplier applied to the controllability of waves, elasticity, and tangential Stokes control, SIAM J. Control and Optim, 403 (2001) 777-800.

[9] J.-P. Puel, Application of Carleman Inequalities to Controllability and Inverse problems, Textos de Metodos Matematicos de l'Instituto de Matematica de l'UFRJ, to appear.

[10] M. Yamamoto, J.-P. Puel, Applications de la contrôlabilité exacte à quelques problèmes inverses hyperboliques, C. R. Acad. Sci. Paris, t. 320, Sér. I (1995) 1171-1776. 